NASA Technical Memorandum 87060 AIAA-85-1213

\title{
Application of Computational Fluid Dynamics to Complex Inlet Ducts
}

C.E. Towne

Lewis Research Center

Cleveland, Ohio

and

E.F. Schum

Rockwell International Corporation

He:

Columbus, Ohio

\section{SEP $3 \quad 1985$}

Anste:

UBRAR , BASA

Mapron, nogma

Prepared for the

Twenty-first Joint Propulsion Conference

cosponsored by the AIAA, SAE, ASME, and ASEE

Monterey, California, July 8-10, 1985 


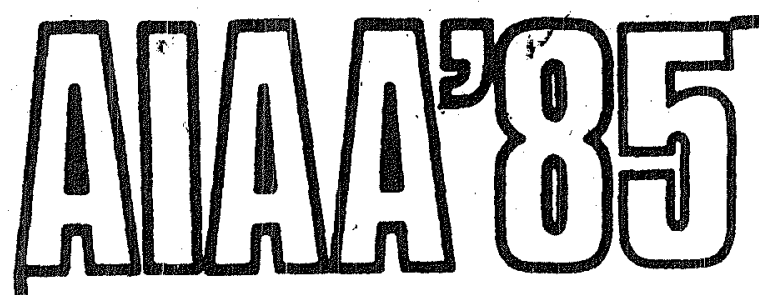

AIAA-85-1213

Application of Computational Fluid Dynamics to Complex Inlet Ducts

C.E. Towne, NASA Lewis Research Center, Cleveland, OH; E.F. Schum, Rockwell International Corporation, Columbus, $\mathrm{OH}$

\section{AIAA/SAE/ASME/ASEE 21st Joint Propulsion Conference July 8-10, 1985 / Monterey California}


C. E. Towne*

NASA Lewis Research Center, Cleveland, Ohio

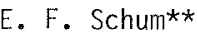

Rockwell International Corporation, Columbus, Ohio

\section{ABSTRACT}

PEPSIG, a 3-dimensional parabol ic Navier-Stokes code, was used to analyze the flow in the subsonic diffuser section of a typical modern inlet design. The effect of curvature of the diffuser centerline and transitioning cross sections was studied to determine the primary cause of flow distortion in the duct. Total pressure values at the engine compressor face are reported.

\section{INTRODUCTION}

High performance military aircraft often utilize inlet designs which are extremely complex because of the highly 3-dimensional nature of the internal flow. In the subsonic diffuser portion of these inlets, there may be changes in the duct cross section coupled with curvature in the duct centerline. Curvature induces cross stream pressure gradients, causing the formation of vortices. When combined with vi scous effects, these pressure gradients can induce regions of 1ocal separation, thereby affecting engine performance. For this reason, it is necessary to be able to accurately predict the flow in these inlets to ensure engine/inlet compatibility for a wide range of flight conditions.

Several approaches can be considered for analyzing the flow in 3-dimensional ducts. Inviscid/boundary layer solutions are no longer adequate to describe the flow since the thickness of the boundary layer can occupy a major portion of the duct cross section. EuTer solutions provide a velocity field but, in the absence of viscosity, can not account for the viscous pressure losses. These losses have a significant effect on engine performance. Fully elliptic Navier-Stokes solutions can provide an accurate flow field, but usually at the expense of hours on today's super computers. In addition, the number of grid points that can be efficiently analyzed is limited by computer storage for fully elliptic solutions. Computer time is of importance in preliminary design analyses where it is necessary to investigate the performance of many inlet configurations and operating conditions. Parabolic spatial marching solutions, on the other hand, afford a considerable reduction in computer time by using a "one-time march" through the duct. In some parabolic solutions of curved duct flow, downstream pressure effects are not transmitted upstream due to the parabolic nature of the solution. What is

\section{* Aerospace Engineer, AIAA Member}

**Member Technical Staff, AIAA Member needed is a computer program which has the capability of providing an elliptic pressure field coupled with a fast parabolic marching, viscous solution. One such method, designated PEPSIG, was developed under the direction of NASA Lewis for accomplishing this (e.g., References 1-3).

It is the purpose of this paper to demonstrate that the PEPSIG program can be used as a cost effective tool for analyzing the viscous flow in complex shaped ducts. Secondary flow and the resulting distortion patterns for several duct configurations are analytically investigated.

\section{APPROACH}

\section{Analysis Method}

The PEPSIG analysis is designed to compute subsonic, compressible flow in 3 mdimensional ducts in which there is little or no streamwise flow separation. Assumptions are made in the algorithm which afford an order of magnitude reduction in computer time when compared with fully elliptic, Navier-Stokes methods. First, it is assumed that the flow is primarily in the direction of the duct centerline, and cross flow velocities are relatively small. This allows a second assumption that the second derivatives in the primary flow direction are negligible. The third assumption is that the pressure in the primary flow momentum equation can be represented by the sum of a 3-dimensional pressure field obtained from a potential flow solution, and a 1-dimensional streamwise pressure correction involving the frictional and viscous mixing effects. The 3-dimensional pressure field is calculated prior to the parabolic march through the duct. It is the third assumption that affords the reduced computer time since only one sweep is needed. The derivation of the governing differential equations and their method of solution have been presented elsewhere (e.g., References 1-3).

Oniy a minimum amount of input data is required, consisting of duct geometry and the flow conditions at the inlet. Duct cross-sectional shapes are represented by superellipse equations, relating the major and minor axis and a corresponding exponent in the ellipse equation. Variation of the duct cross-sectional shape in the flow direction is accounted for by representing the major axis, minor axis, and the exponent as polynomial functions of distance in the flow direction. The curved centerline is also described by poiynomials. Other input data include the inlet Reynolds number, Mach number, 
and the initial boundary layer thickness. The code contains its own grid generation routine.

\section{Program Validation}

Confidence in the use of a program is best gained by comparing analytical results with experimental data. This has been done for the PEPSIG analysis in References 2-5. Other PEPSIG results are shown in Reference 6 . As an example, in this paper results will be shown for turbulent flow in a circular cross section duct that has an S-shaped centerline (Reference 4 ). This is of particular interest because most engine inlets operate in the turbulent flow regime, and the inlets often have $S$-shaped centerlines. The test configuration consisted of two 22.5 degree, circular arc bends. The diameter, $D$, was $48 \mathrm{~mm}$, and the centerline radius, $R$, was $336 \mathrm{~mm}$, for an $R / D$ of 7 . The Reynol ds number based on diameter and average inlet velocity was 48,000 , and the corresponding Dean number was 12,828 . The Dean number is the product of the Reynolds number and the square root of the duct radius/radius of centerline curvature.

Computed secondary velocities near the inflection and exit planes are shown in Figure 1. In Figure 2 the predicted and measured streamwise velocities are compared. The more dense mesh (50 $x 50$ ) in the cross stream plane improved the agreement, as expected.

\section{Duct Configurations and Inlet Conditions}

Three basic geometric configurations were examined in the present study, as shown in Figure 3. The first, called the baseline configuration, represents a typical modern inlet design with a curved centerline and a transitioning crosssectional shape. The cross section is represented by a superellipse, and transitions from nearly rectangular at the inlet to circular at the exit. The other two configurations are derived from the first, and were used to isolate the effects of cross section transitioning and centerline curvature on the flow. The second configuration has the same distribution of cross section shape, but with a straight centerline. The third configuration has the same centerline shape and area distribution as the baseline configuration, but with a circular cross section.

Conditions assumed at the diffuser inlet included a total pressure of 800 psf and a Mach (M) number of 0.5 . This corresponds to flight at about 28,000 feet altitude. A turbulent boundary layer thickness equal to 4.8 percent $(\delta=.048)$ of the duct half width was also assumed for the baseline and straight centerline configurations. For the circular cross section configuration, a thickness of 0.056 was used to give the same initial blockage. Effect of boundary layer thickness and Mach number on the computed results was investigated.

\section{RESULTS AND DISCUSSION}

The effect of an S-shaped duct on flow distortion is first discussed for a circular cross- sectional contour, Figure $3 c$. The combined effect of an S-shaped duct with transitioning cross sections, Figure $3 a$, is then discussed. Lastly, the flow in a straight duct with transitioning cross sections is discussed.

\section{S-Shaped Duct with Circular Cross Sections}

Streamwise velocity contours, cross stream velocities and total pressure contours are presented in Figures 4-6. For clarity, only $1 / 4$ of the actual number of grid points used are shown in the cross stream velocity plot. Total pressure contours are based on the ratio of the local total pressure to that at the first station.

The physics behind the flow patterns in S-shaped ducting can be best described by examining the cross stream velocities, Figure 5 . At the first station, Figure $5 a$, cross stream velocities are smal1. The effect of the first bend on the flow pattern can be seen at the station $b$. The core of the flow moves away from the center of curvature of the duct due to the centrifugal forces. Due to this curvature, the local pressure decreases toward the center of curvature causing the low energy fluid near the wall and in the thin boundary layer to move toward the lower pressure region. A vortex motion starts to form but is quickly dissipated when the cross flow pressure gradients reverse in the second bend, Figure $5 \mathrm{c}$. At this station, the boundary layer reverses its direction as it flows toward the lower pressure region, now at the left side of the duct. By station d (inflection point between second and third bends) a pair of counter rotating vortices has formed. These vortices, which persist into the third bend (1ocations $d$ and e), move the low energy fluid along the sides of the wall. At station e the cross flow pressure gradients have reversed direction again, causing the formation of two pairs of counter rotating vortices at the top and bottom. These two pairs interact, driving the low energy flow away from the wall, at stations $e$ and $f$. This viscous interaction reduces the local total pressures at $e$ and $f$, Figure 6 . This vortex action causes a corresponding bulge in the streamwise velocities, Figures $4 \mathrm{e}-\mathrm{f}$.

A small separation bubble is formed at station $c$ in Figures 4-6, near the surface on the right. The flow reversal is caused by a local adverse pressure gradient resulting from the change of wall curvature from convex (low pressure) to concave (high pressure). This situation is probably aggravated by the movement of low energy flow toward the inside location of the duct in the first bend. These results demonstrate that PEPSIG can march through a moderate flow reversal region without "bombing out". This is a desirable feature because, if the program stops, one would not know that the separation bubble is small. A small region of reverse flow may be tolerable or can be eliminated by a simple change in the duct shape. The FLARE approximation (Reference 7) is used in the calculations in the flow reversal region. In this, an approximation is made to the density-velocity-velocity gradient term, $\rho u \partial u / \partial x$, in the streamwise momentum equation. When the 
velocity is negative, it is replaced by a small positive value and the marching continued. The CPU time for this case was 7.7 minutes on the NASA LEWIS CRAY-1.

\section{Baseline Configuration}

Streamwise velocity contours, cross stream velocities and total pressure profiles are shown in Figures 7-9. Overall trends are identical to that for the $S$-shaped duct with a circular cross section, shown in Figures 4-6. This shows that the physics of the flow in the two ducts is basically the same. Counter rotating vortices are al so formed at station b, Figure 8, along the top and bottom surfaces. Two pairs of counter rotating vortices occur at location $d$ near the corners on the right side. The intensity of these vortices decreases toward the end of the duct. Total pressure profiles at the engine compressor face, Figure $9 \mathrm{f}$, are about the same as for the $S$-shaped duct with the circular cross section, Figure $6 f$.

A separation bubble is also formed at station b (Figure 8) along the surface on the right which is dissipated by station $C$. The CPU time for this run was 8.3 minutes on the CRAY -7 .

In the analysis of the baseline configuration, a total of 262,500 grid points was used, $50 \times 50$ in the cross plane at each of 105 flowwise stations. To ensure that the grid density was adequate to provide sufficient resolution of the boundary layer and secondary flow patterns, the number of grid points was increased in the cross plane and in the flowwise direction. Figure 10 presents the streamwise velocity contours for the case where the number of cross plane grid points was increased four fold to $100 \times 100$. The results are about the same as for the baseline $50 \times 50$ grid density, shown in Figure 7 . The CPU time was 38.3 minutes. Similar agreement was obtained when the number of spanwise stations was increased to 209 from the baseline value of 105, as shown in Figure 11. Computer running time for this case was 15.6 minutes. In both cases of increased grid density, the total pressure recovery at the engine compressor face is about the same as for the baseline, Figure 9 . These results show that the $50 \times 50 \times 105$ grid for the baseline configuration is adequate to describe the flow phenomena.

To determine the sensitivity of the results to the thickness of the boundary layer at the inlet, the value used in the baseline analysis, $\delta=0.048$, was arbitrarily increased by a factor of 5. Isovels and corresponding total pressure contours are shown in Figures 12 and 13. The basic flow phenomena are the same even though the boundary layer along the duct is much thicker, shown by the total pressure contours. It should be noted, however, that there is a significant reduction in the total pressure recovery at the engine face, Figure $13 \mathrm{f}$, when compared to the basel ine, Figure $7 f$. The inlet boundary layer must be minimized to maximize the total pressure recovery at the engine face.
Results are presented in Figure 14 in which the baseline inlet Mach number was reduced from 0.5 to 0.01 , an extreme case. Overa11 trends are the same as in the previous cases.

\section{Straight Duct With Transitioning Cross Sections}

To confirm that the primary cause of the flow distortion and total pressure losses is due to the $S$-shape ducting, the base configuration was modified to be straight, Figure 3b. Streamwise velocity and total pressure contours are shown in Figures 15 and 16. There is no significant distortion with the straight duct even with the transitioning cross section.

\section{CONCLUDING REMARKS}

For the diffuser configurations and inlet conditions studied, it was shown that:

- PEPSIG is an efficient analytical design tool for calculating the flow in complex shaped ducts. CPU times are the order of 8 minutes on the CRAY- 1 for 262,500 grid points as compared to hours normally required in fully elliptic solutions.

- Curvature of the duct centerline and not transitioning cross sections is the primary cause of the flow distortions and total pressure losses for the configurations studied.

- Thickness of the boundary layer at the diffuser inlet should be kept as thin as possible to minimize the total pressure losses in the ducts.

\section{REFERENCES}

1. Briley, W. R., and McDonald, H.: "Analysis and Computation of Viscous Subsonic Primary and Secondary Flows," AIAA Paper 79-1453, July 1979.

2. Levy, R., McDonald, H., Briley, W. R., and Kreskovsky, J. P.: "A Three-Dimensional Turbulent Compressible Subsonic Duct Flow Analysis for Use with Constructed Coordinate Systems," AIAA Paper 80-1398, July 1980.

3. Levy, R., Briley, W. R., and McDonald, h.: "Viscous Primary/Secondary Flow Analys is for Use with Nonorthogonal Coordinate Systems," AIAA Paper 83-0556, Jan. 1983.

4. Towne, C. E.: "Computation of Viscous Flow in Curved Ducts and Comparison with Experimental Data," AIAA Paper 84-0531, January 1984.

5. Anderson, B. H.: "Three-Dimensional Viscous Design Methodology for Advanced Technology Aircraft Supersonic Inlet Systems," NASA TM 83558, AIAA Paper 84-0192, January 1984.

6. Vakili, $A_{*}$, Wu, J. M., Hingst, W. R., and Towne, C. E.: "Comparison of Experimental and Computational Compressible Flow in an S-Duct," AIAA Paper 84-0033, Jan. 1984.

7. Reyhner, T. A., and Flugge-Lotz, I.: "The Interaction of a Shock Wave with a Laminar Boundary Layer", International Journal of Non-Linear Mechanics, Vol. 3, 1968 , pp. 173-199. 


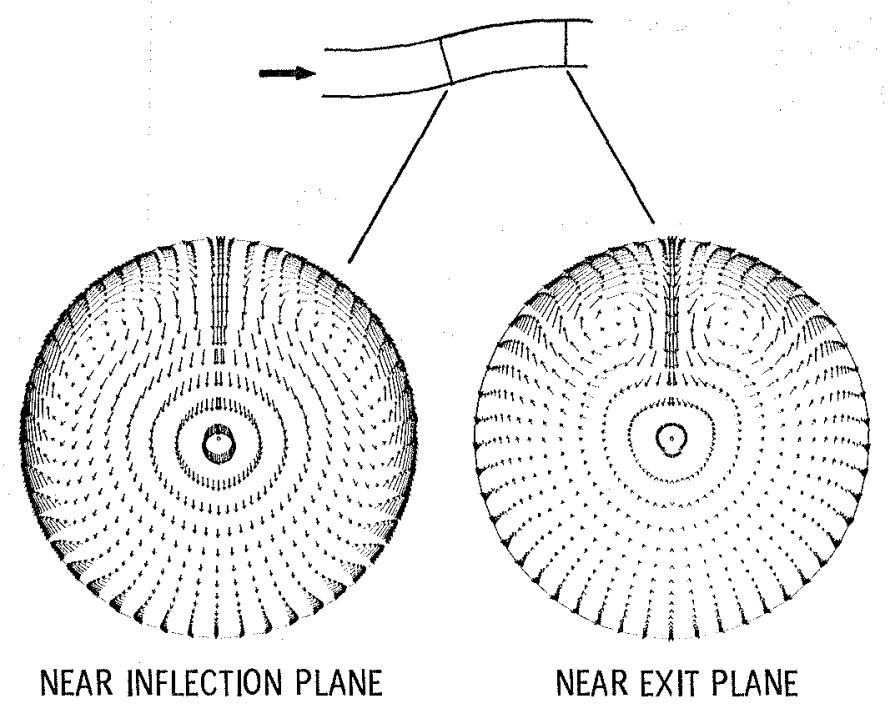

Figure 1. - Computed secondary velocities for turbulent flow in 22. 5-22. $5^{\circ}$ circular S-duct.

\section{EXPERIMENT}

ANALYSIS, 50×50×80 GRID

ANALYSIS, $25 \times 25 \times 80$ GRID

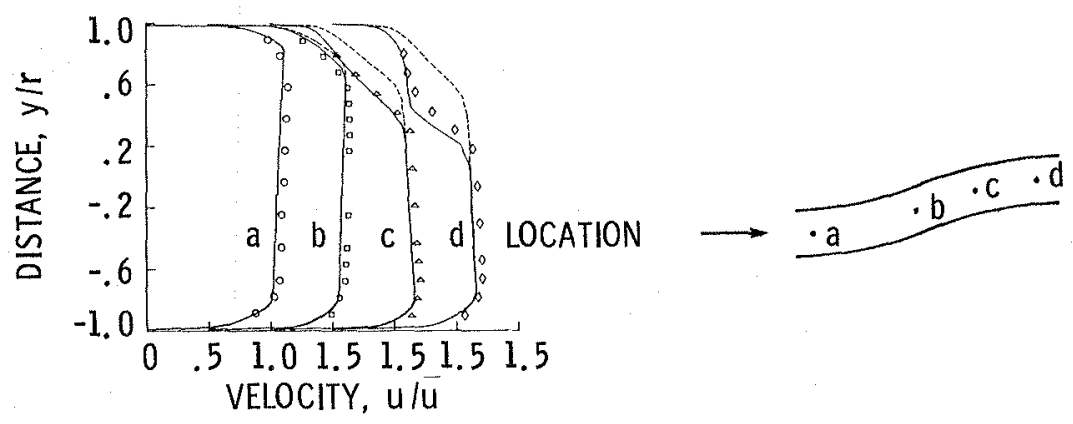

Figure 2. - Computed and experimental streamwise velocity profiles in symmetry plane for turbulent flow in $22.5-22.5^{\circ}$ circular $S-$ duct. 


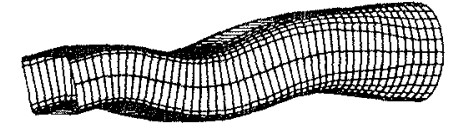

(a) Baseline.

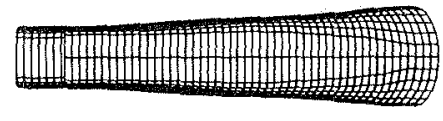

(b) Straight centerline.

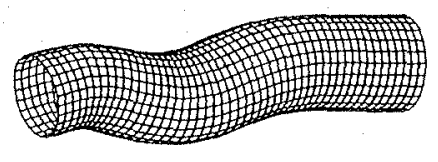

(c) Circular cross-section

Figure 3. - Inlet configurations.

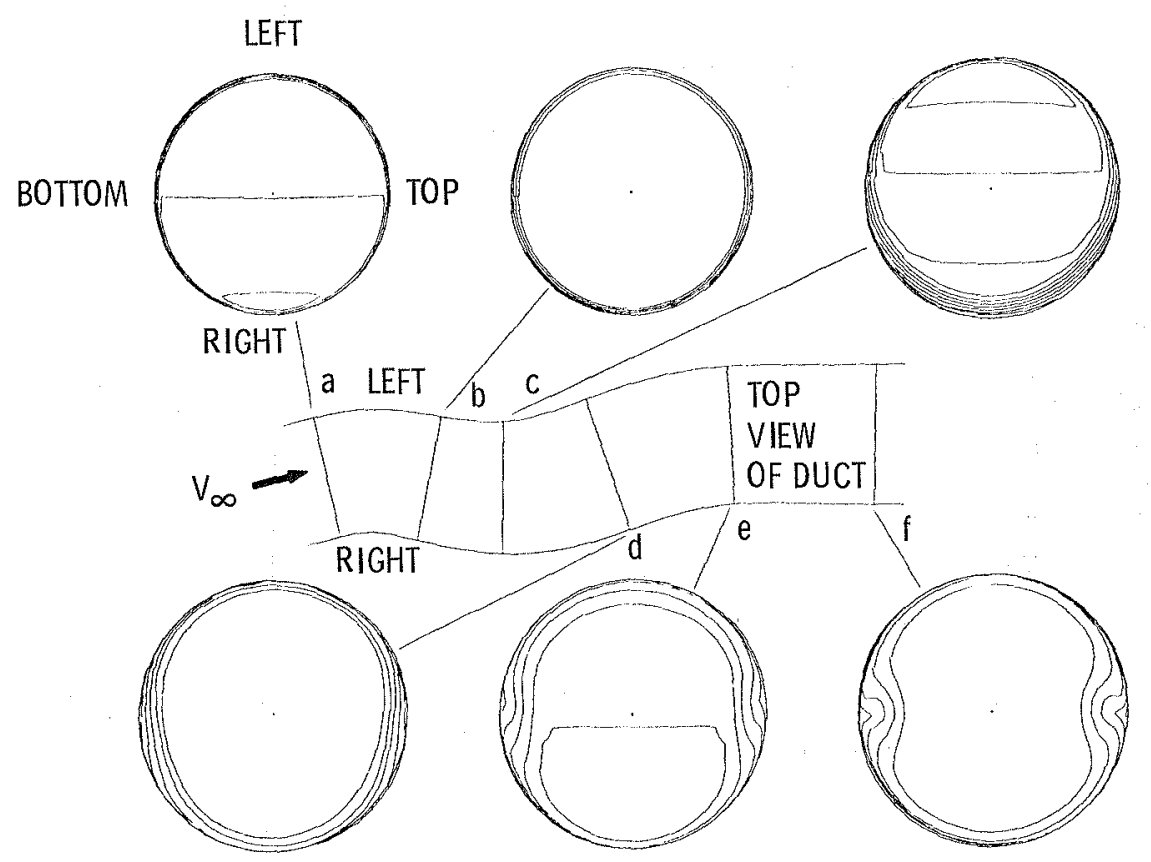

Figure 4. - Computed streamwise velocity contours in circular cross-section configuration, $M=0.5, \delta=0.056,50 \times 50 \times 105$ mesh. 




Figure 5. - Computed cross stream velocities in circular cross-section configuration, $M=0.5$, $\delta=0.056,50 \times 50 \times 105$ mesh. 


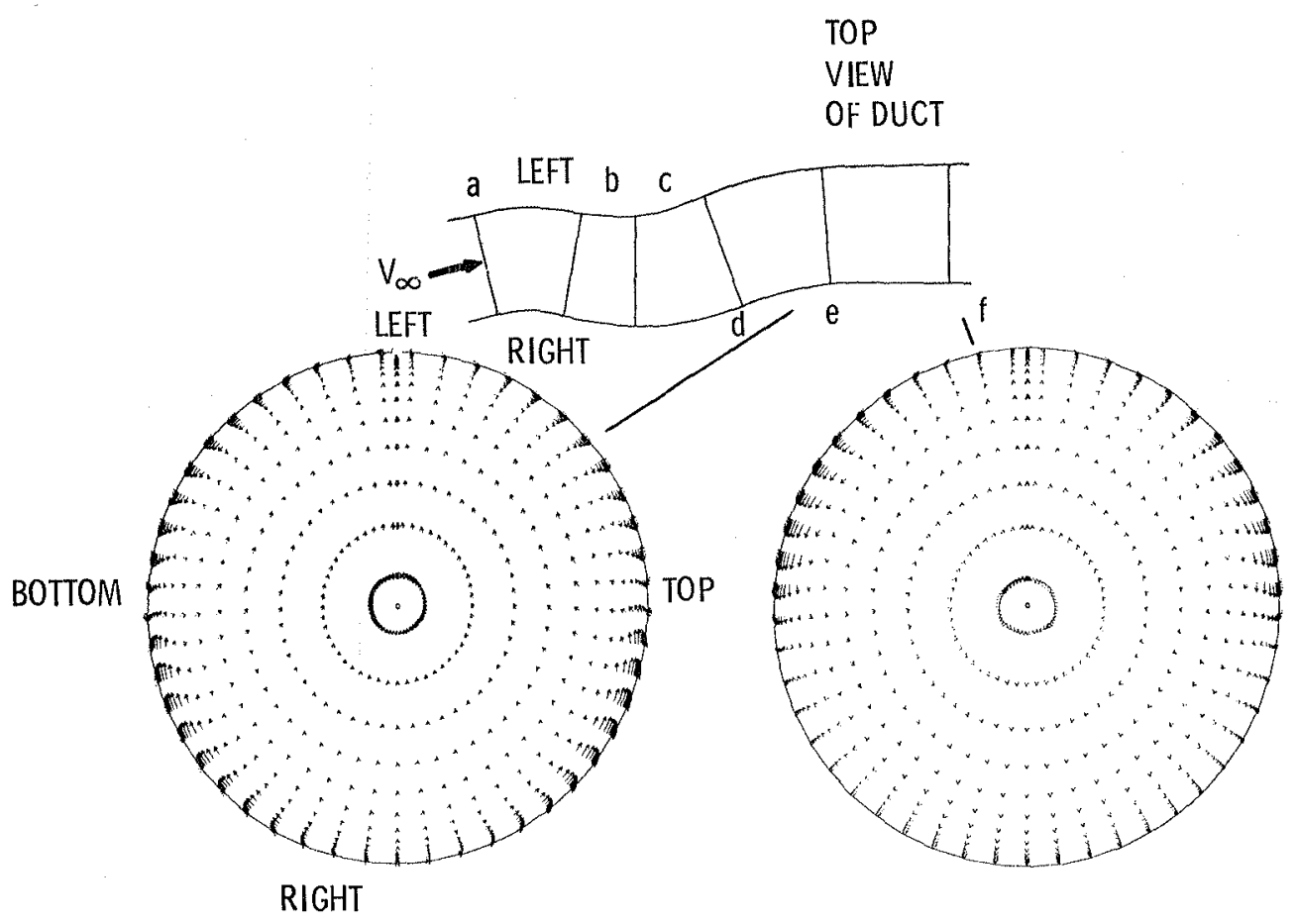

Figure 5. - Concluded.

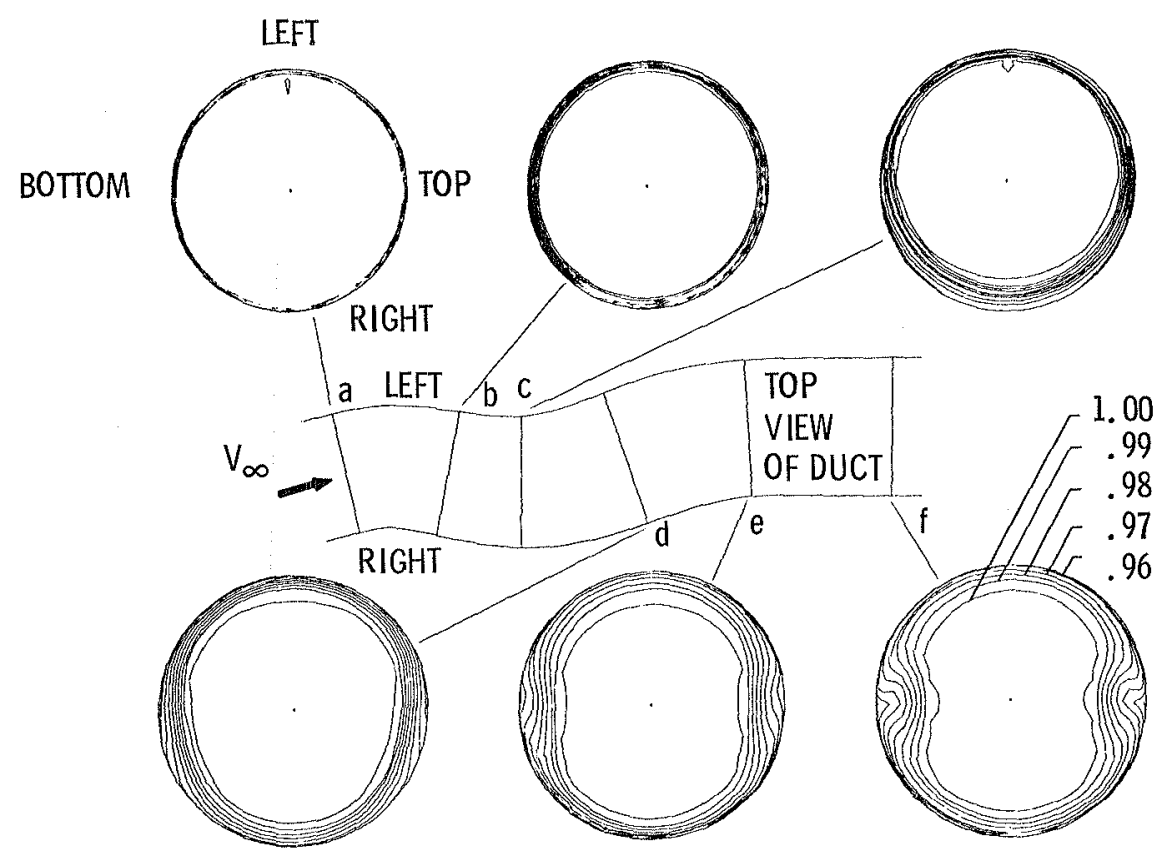

Figure 6. - Computed total pressure contours in circular cross-section configuration, $M=0.5, \delta=0.056,50 \times 50 \times 105$ mesh. 


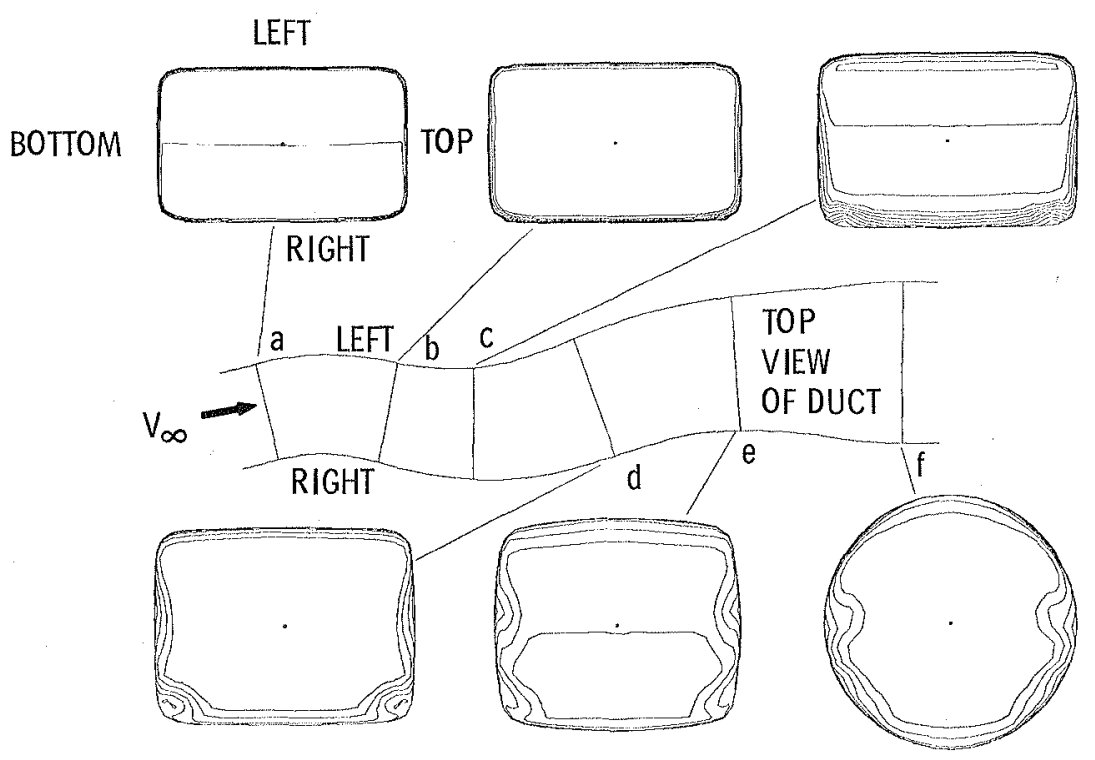

Figure 7. - Computed streamwise velocity contours in baseline configuration, $M=0.5, \delta=0.048,50 \times 50 \times 105$ mesh.

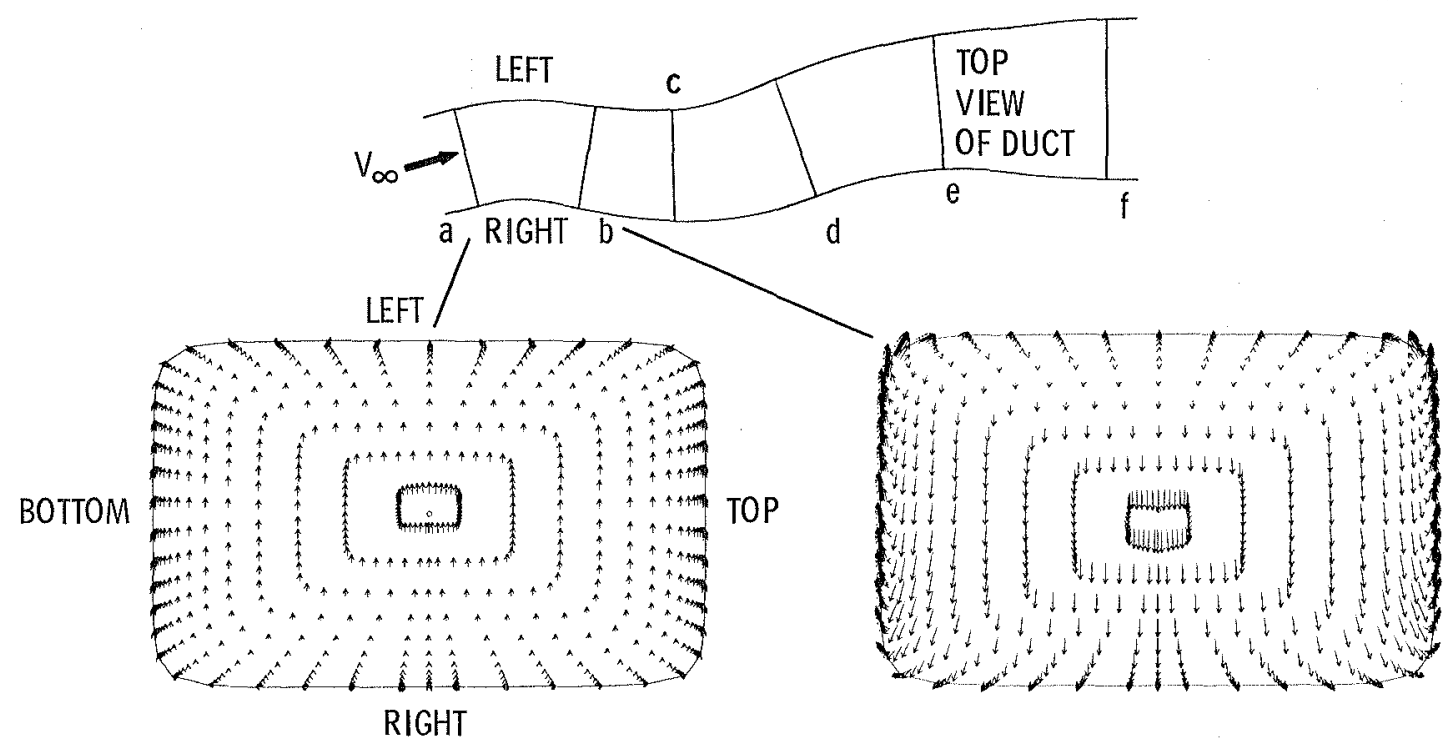

Figure 8. - Computed cross stream velocities in baseline configuration, $M=0.5, \delta=0.048,50 \times 50 \times 105$ mesh. 


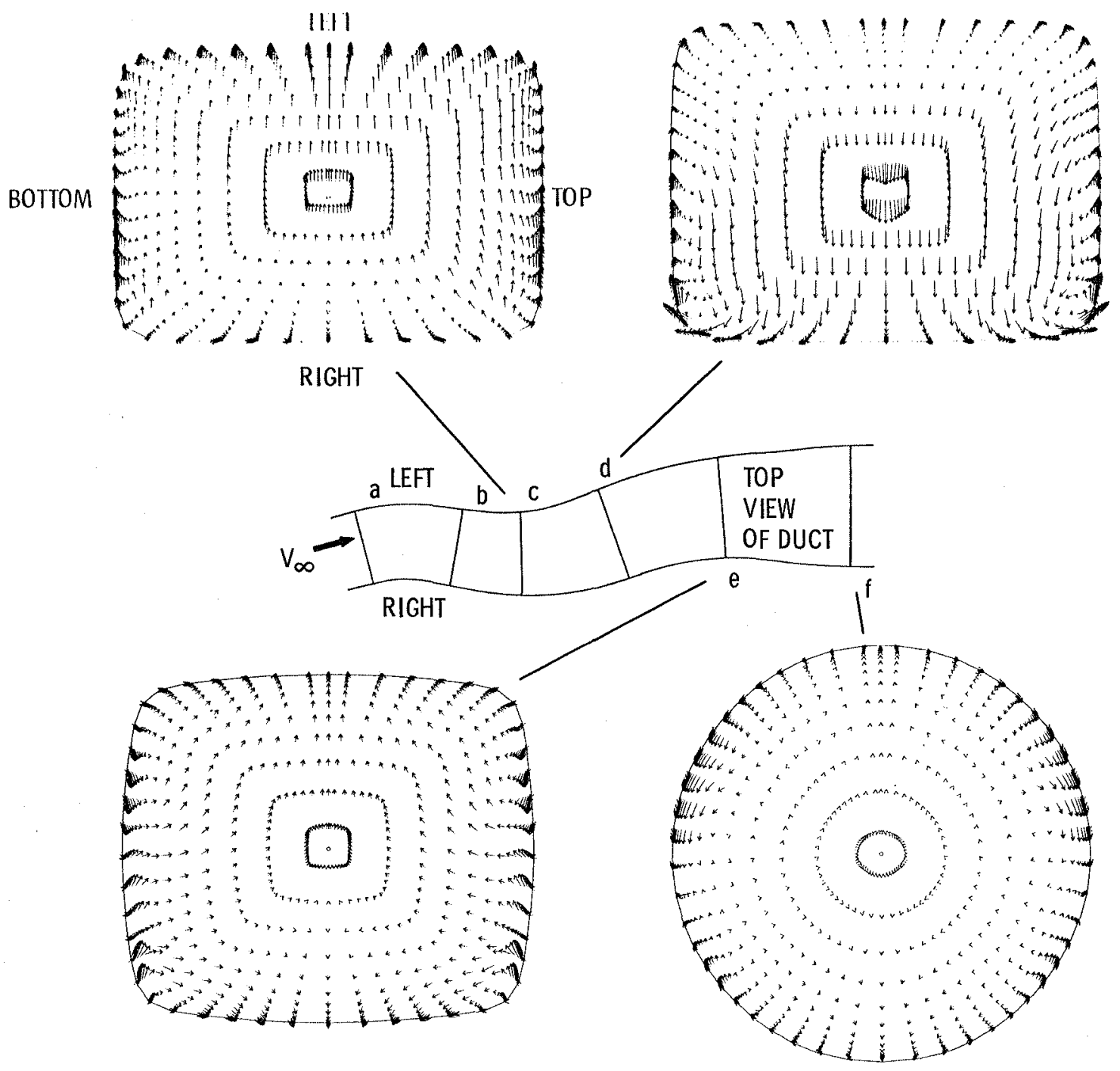

Figure 8. - Concluded. 


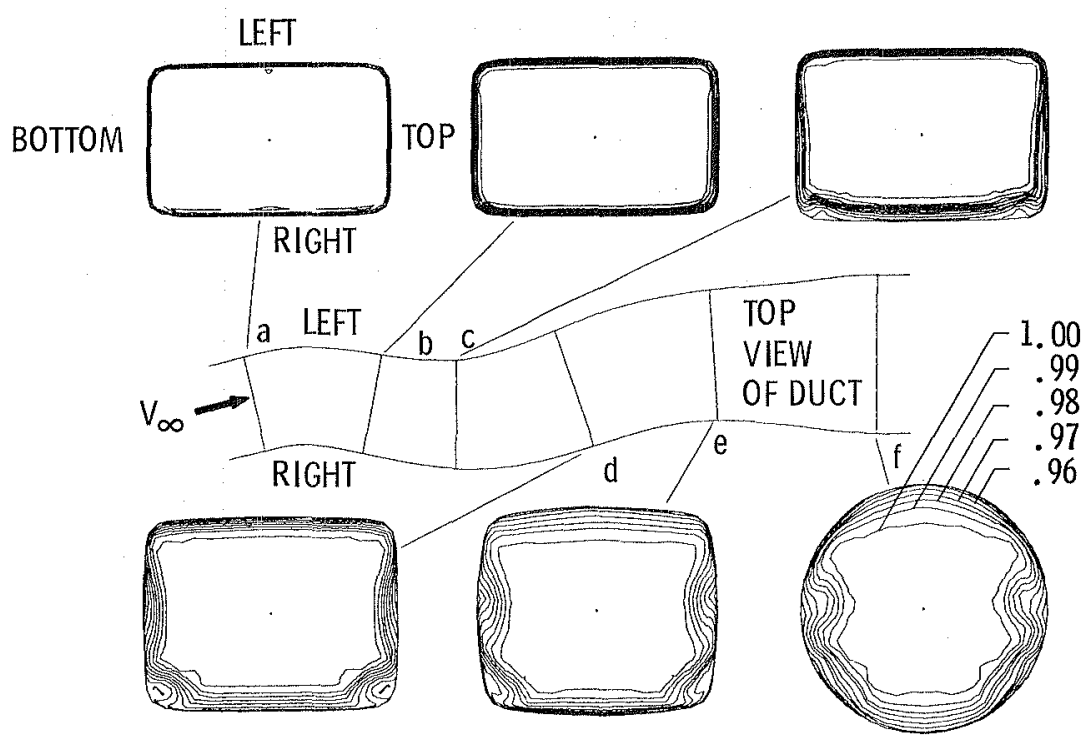

Figure 9. - Computed total pressure contours in baseline configuration, $M=0.5, \delta=0.048,50 \times 50 \times 105$ mesh.

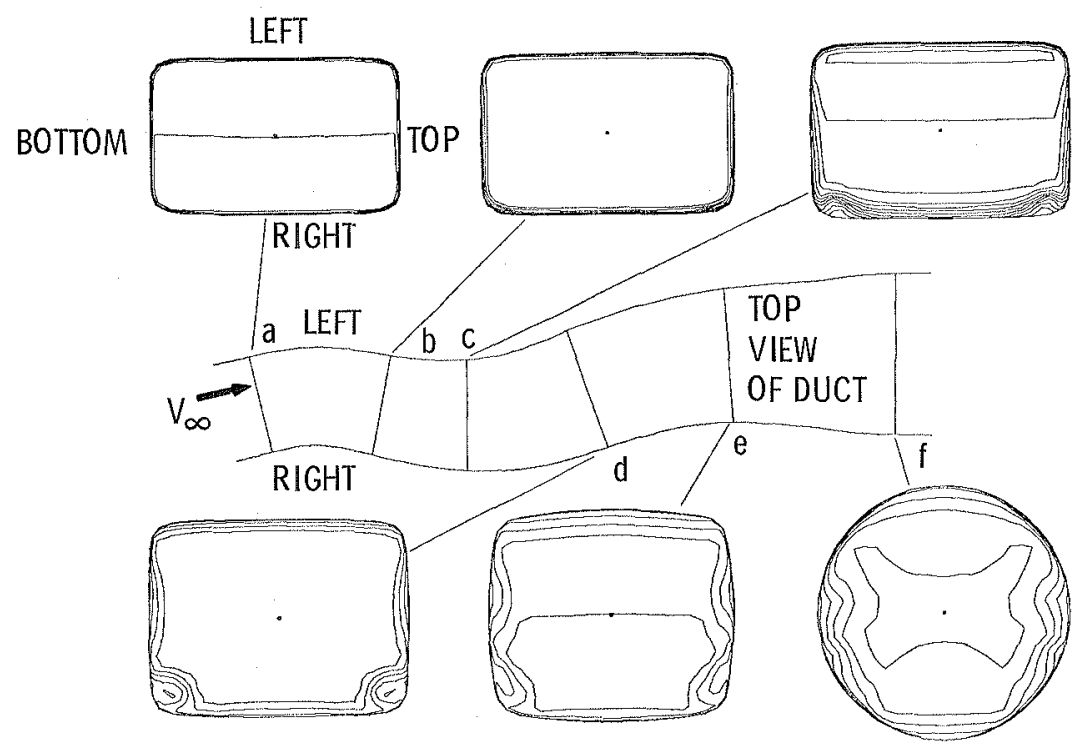

Figure 10. - Computed streamwise velocity contours in baseline configuration, $M=0.5, \delta=0.048,100 \times 100 \times 105$ mesh. 


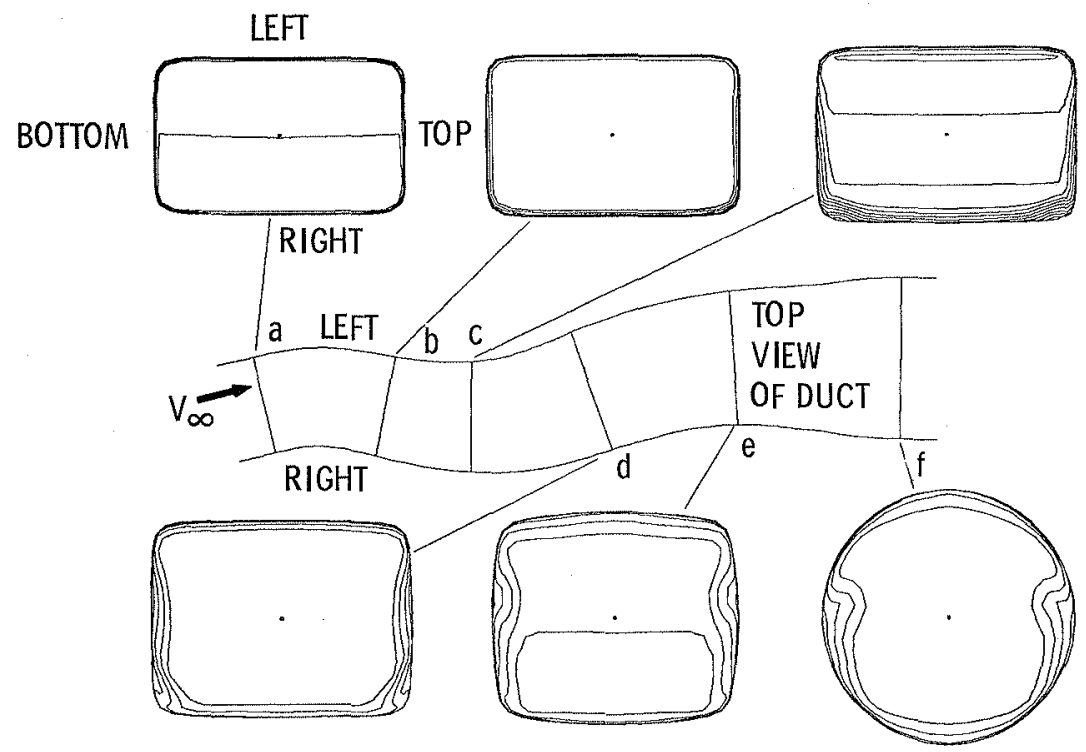

Figure 11. - Computed streamwise velocity contours in baseline configuration, $M=0.5, \delta=0.048,50 \times 50 \times 209$ mesh.

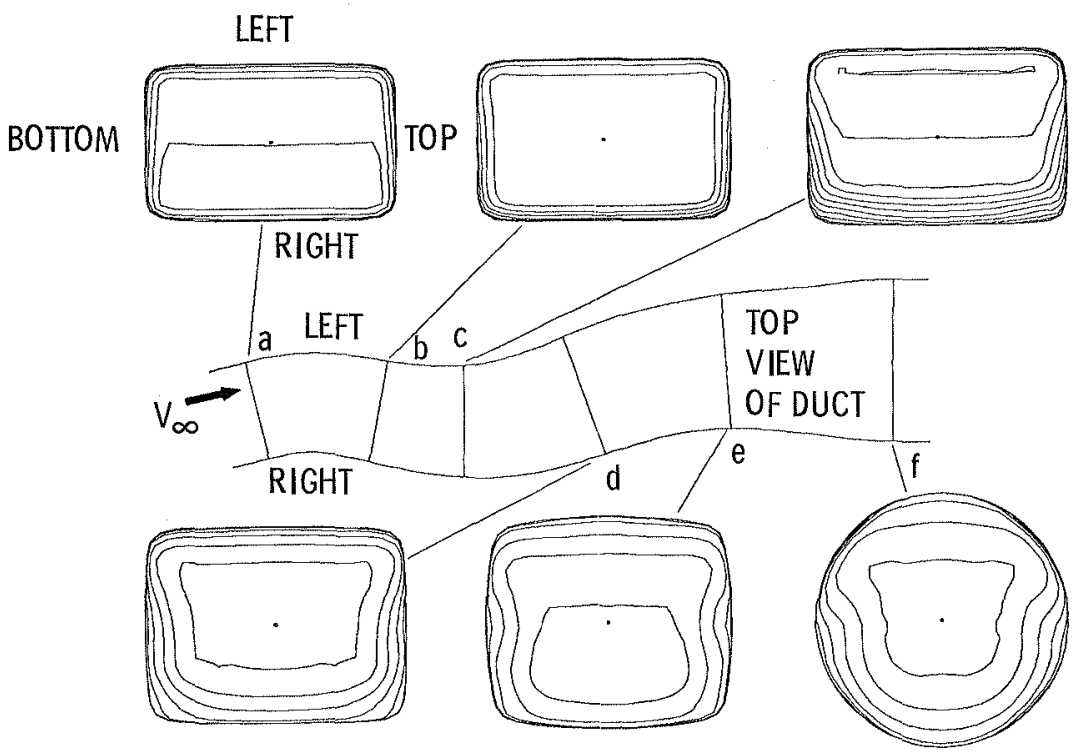

Figure 12. - Computed streamwise velocity contours in baseline configuration, $M=0.5, \delta=0.24,50 \times 50 \times 105$ mesh. 


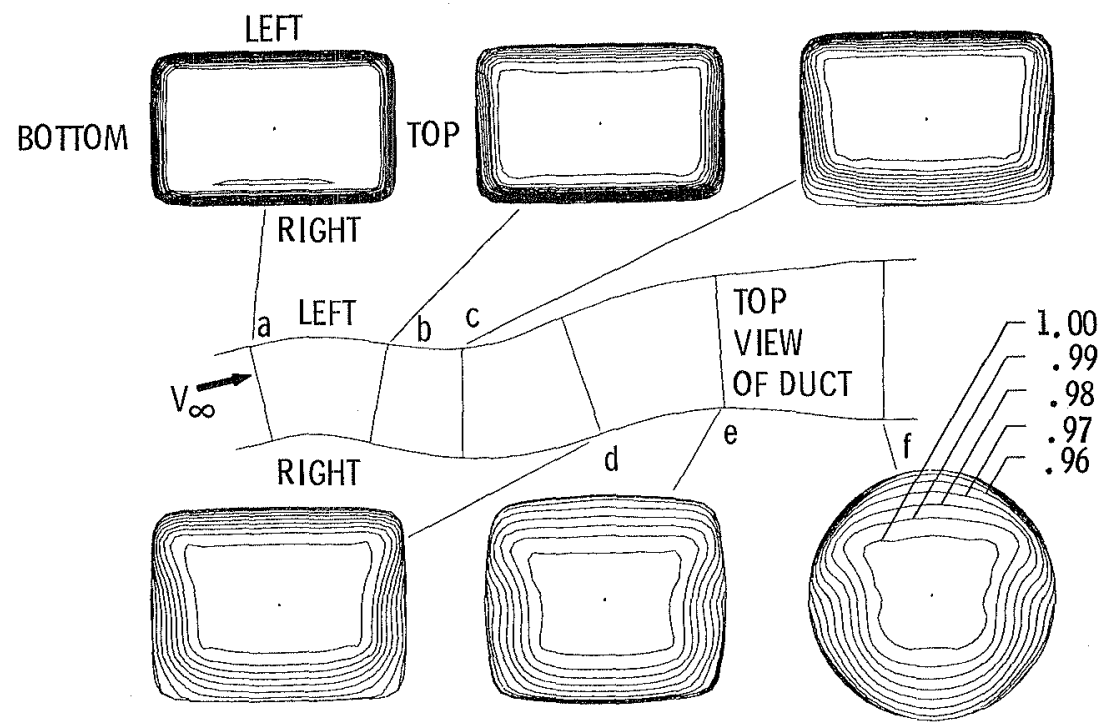

Figure 13. - Computed total pressure contours in baseline configuration, $M=0.5, \delta=0.24,50 \times 50 \times 105$ mesh.

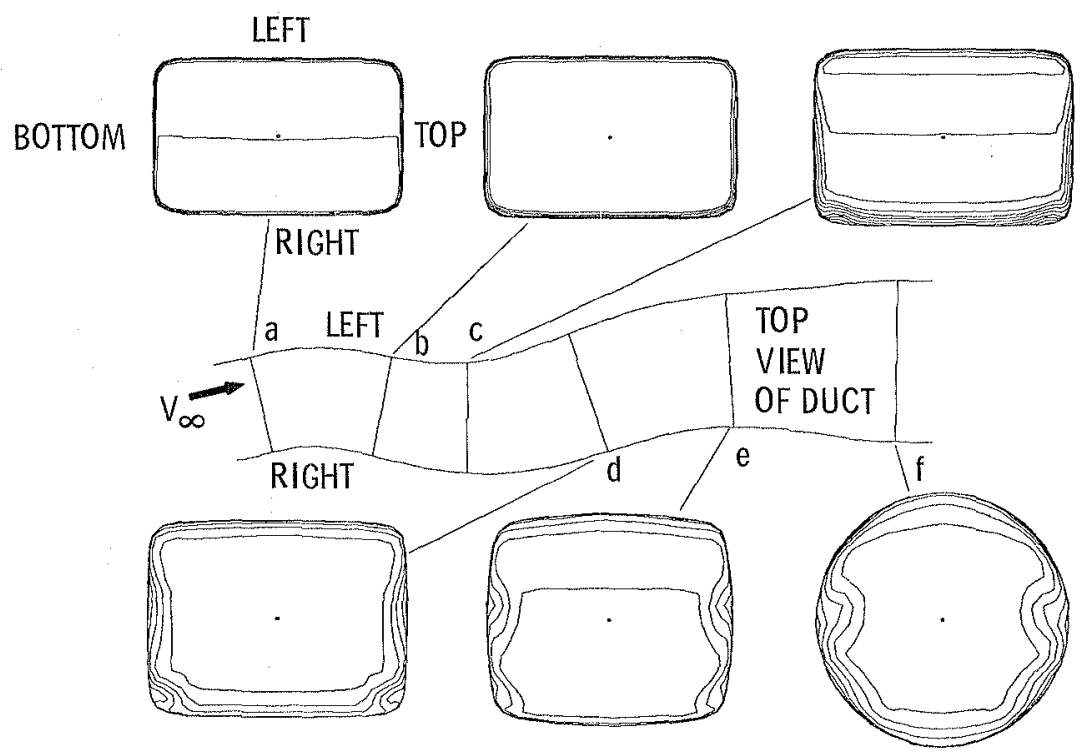

Figure 14. - Computed streamwise velocity contours in baseline configuration, $M=0.01, \delta=0.048,50 \times 50 \times 105$ mesh. 


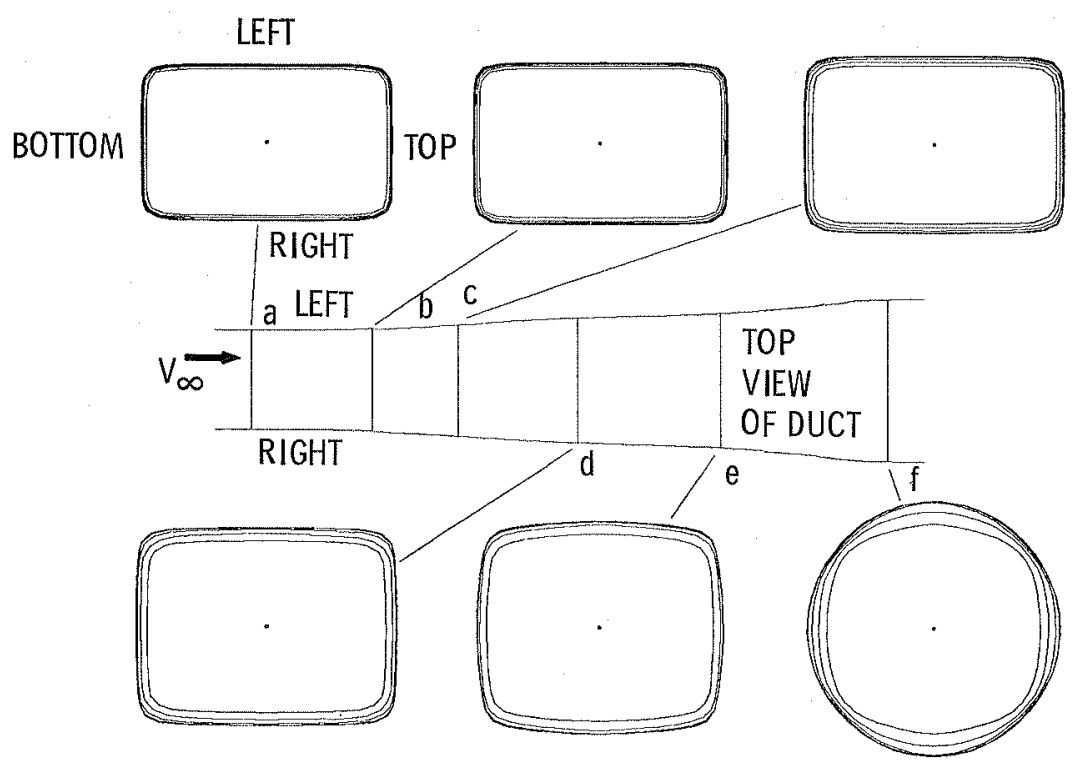

Figure 15. - Computed streamwise velocity contours in straight centerline configuration, $M=0.5, \delta=0.048,50 \times 50 \times 105$ mesh.

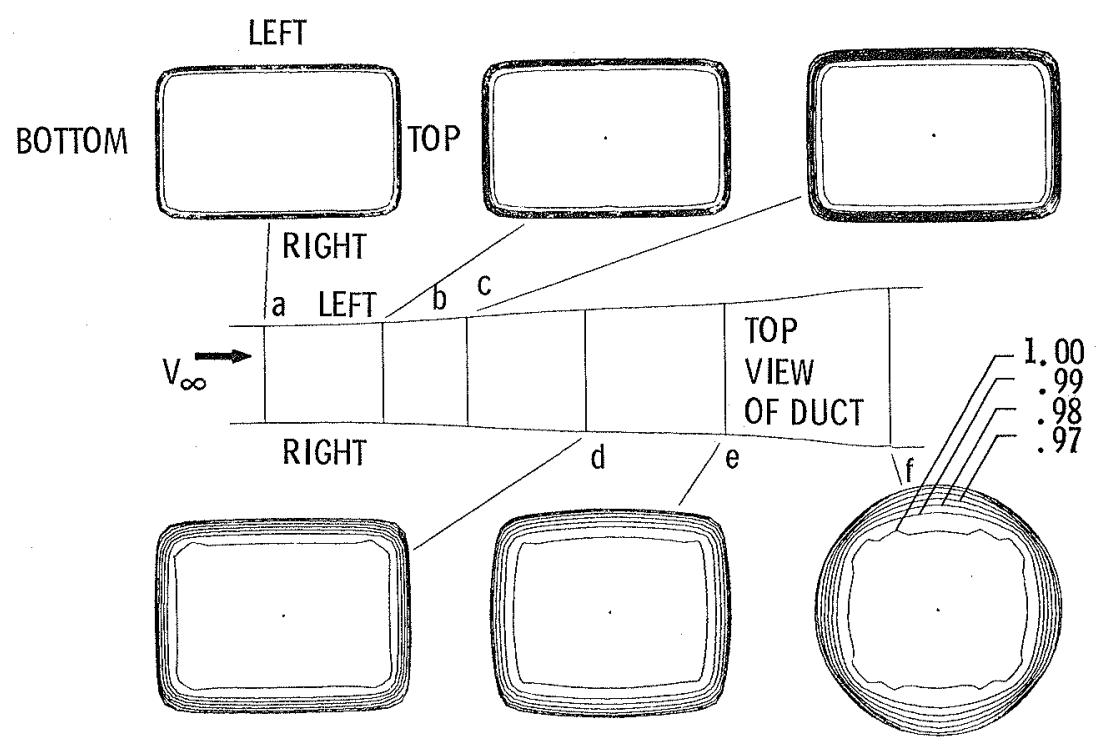

Figure 16. - Computed total pressure contours in straight centerline configuration, $M=0.5, \delta=0.048,50 \times 50 \times 105$ mesh. 


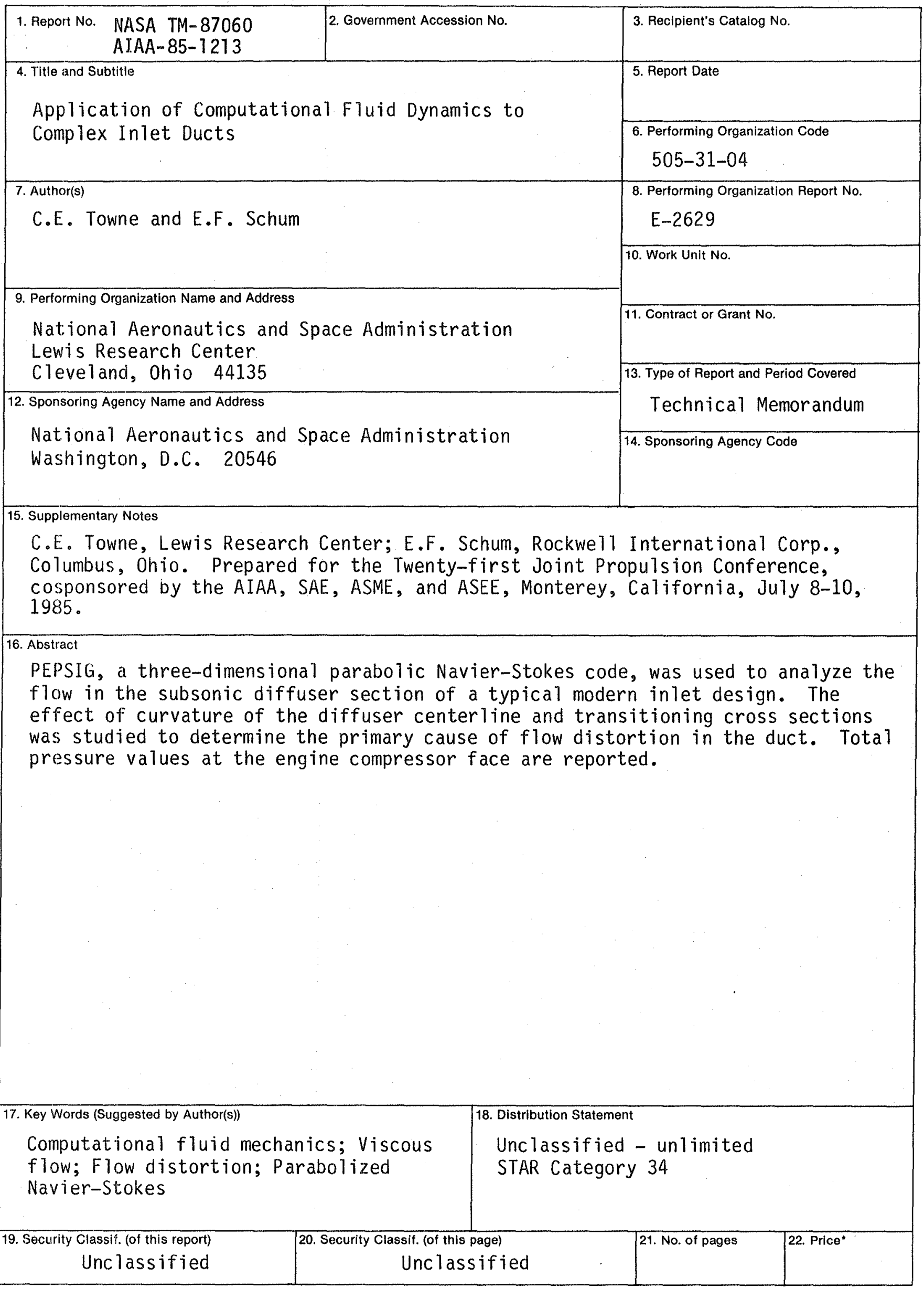

${ }^{*}$ For sale by the National Technical Information Service, Springfield, Virginia 22161 


\section{End of Document}

